\title{
The Middle Turbinate Resection and Its Repercussion in Olfaction with the University of Pennsylvania Smell Identification Test (UPSIT)
}

\author{
Fernando Cesar Mariano ${ }^{1,2}$ Rogerio Hamerschmidt ${ }^{3}$ Caio Marcio Correa Soares ${ }^{4,5}$ \\ Ana Tereza Moreira 6 \\ ${ }^{1}$ Surgery Department, Complexo Hospital de Clínicas da Universidade \\ Federal do Paraná (CHC-UFPR), Curitiba, PR, Brazil \\ 2 Instituto Paranaense de Otorrinolaringologia (IPO), Curitiba, \\ PR, Brazil \\ ${ }^{3}$ Department of ENT, Instituto Paranaense de Otorrinolaringologia \\ (IPO), Curitiba, PR, Brazil \\ ${ }^{4}$ Education and Research Center, Hospital Paranaense de \\ Otorrinolaringologia, Curitiba, PR, Brazil \\ ${ }^{5}$ Department of Otorhinolaryngology, Setor de Ciências da Saúde da \\ Universidade Federal do Paraná (UFPR), Curitiba, PR, Brazil \\ ${ }^{6}$ Department of Ophthalmology, Universidade Federal do Paraná \\ (UFPR), Curitiba, PR, Brazil \\ Int Arch Otorhinolaryngol 2018;22:280-283. \\ Address for correspondence Fernando Cesar Mariano, MD, \\ Departamento de Cirurgia, Complexo Hospital de Clínicas da \\ Universidade Federal do Paraná (CHC-UFPR), Rua General Carneiro, \\ 181, Curitiba, PR, 80060-900, Brazil (e-mail: fcrmariano@gmail.com).
}

\begin{abstract}
Keywords

- olfaction

- turbinates

- nasal obstruction

Introduction Nasal obstruction is a common complaint, and, for some, the middle turbinate resection is still a controversial issue among the surgical options due to the possibility of deleterious effects on olfaction. The University of Pennsylvania smell identification test (UPSIT) is considered the gold standard of smell identification tests, but data about it is still incipient in Brazil.

Objective To evaluate if the middle turbinectomy has any repercussion on the sense of olfaction by using the UPSIT as an assessment tool.

Methods A prospective study performed between 2013 and 2015 with 27 patients who were treated with middle turbinectomy by the same surgeon and tested with the UPSIT pre- and post-surgery, with a minimum interval of 3 months.

Results Twenty-five patients completed the study. The mean age was 27.9 years. There was no statistical correlation between middle turbinectomy and the UPSIT score, or between gender and the UPSIT score.

Conclusion There was no clinical repercussion on olfaction from partial middle turbinectomy.
\end{abstract}

\section{Introduction}

People can live for 40 days without food and 4 days without water, but can only survive for 4 minutes, at most, without breathing. ${ }^{1}$ This medical aphorism synthesizes the importance of respiration and, as the nose is responsible for $70 \%$ of the total airway resistance, ${ }^{2}$ mastering the surgical techniques available to increase nasal patency, including the middle turbinectomy, is essential for otolaryngologists. A healthy sense of olfaction guarantees quality of life by preserving the ability to experience flavor and thus, to derive pleasure from gastronomy. It is also essential for maintaining self-confidence and good social received

September 7, 2017

accepted

September 30, 2017

published online

December 6, 2017
DOI https://doi.org/

10.1055/s-0037-1608679.

ISSN 1809-9777.
Copyright $\odot 2018$ by Thieme Revinter

Publicações Ltda, Rio de Janeiro, Brazil
License terms

(c) $(1) \$$ 
relationships as well as for keeping the home safe by alerting to possible fire situations.

Recent modeling studies have shown that small alterations in nasal anatomy may alter local airflow and turbulence to a much greater extent than they alter total airflow. ${ }^{3}$ Although Clement ${ }^{4}$ showed an increase in the publication of studies on surgery of the middle turbinate in parallel with the development of functional endoscopic sinus surgery (FESS), only a few of these studies focused on the relationship between the middle turbinate resection and the sense of olfaction. ${ }^{5-7}$ There are still controversies about the safety of middle turbinectomy and even less information in a population of patients free of sinusitis, probably with less inflammatory sequelae to its olfactory neuroepithelium.

The patients' subjective assessments of olfactory acuity do not correlate with the results of the smell tests, in general. The fact that Fornazieri ${ }^{8}$ had just concluded the standardization of the smell test (University of Pennsylvania Smell Identification Test [UPSIT]) for the Portuguese language, with proper adaptation to the Brazilian culture, provided an interesting tool for national clinical investigations in multidisciplinary fields (geriatrics, neurology, neurosurgery, besides ear, nose and throat [ENT]). ${ }^{9-11}$

The goal of this study is to evaluate the repercussion of the middle turbinectomy in the olfactory sense by using the UPSIT as an assessment tool, and also to contribute to rhinology in the understanding of the risks and benefits of this procedure.

\section{Methods}

This study was approved by the ethics committee of the institution (23/2013). Twenty-seven patients were selected in a study of a contemporary longitudinal cohort, including both sexes. The patients included in the study had not succeeded in improving nasal obstruction with clinical treatment; thus, they underwent septoplasty and middle turbinectomy with the purpose of ameliorating the nasal patency. This decision was based on the patients' disease process as defined by signs, symptoms and preoperative nasal fiberoptic exam. The foremost sign was the presence of a middle turbinate that appeared large and bulged anteriorly, with a moderate to severe inflammation or suspicion of bullous concha, in a way that overcame the obstruction aspect of the inferior turbinate. They were classified as type 3, according to the classification proposed by Lee, ${ }^{12}$ which is further discussed somewhere else. The inferior turbinates were not operated in any case.
Prior to surgery, all patients signed a free and informed consent form approved by the ethics committee. In order to ascertain olfactory acuity, they took the UPSIT, which consists of four booklets, each with 10 pages, amounting to 40 microencapsulated scratch and sniff odorant strips located at the bottom of each page, and are supposed to be presented to both nostrils simultaneously. The subject releases the odor by scratching the strip with a pencil tip, in a standardized manner. He or she then indicates the smell that is perceived by choosing a name from a set of four odor descriptors located just above the odorized strip. This test scoring is based on the number of correct answers that can range from 0 to 40 , in which 0 represents complete anosmia and 40 represents a perfect score, interpreted according to the individual's age and gender. The patients who stated subjectively that they did not have a normal sense of olfaction, and those with alterations in the computed tomography (CT) scan of the paranasal sinuses, compatible with chronic rhinosinusitis, were not allowed to participate in the study.

Two patients were lost to follow-up.

The middle turbinate resection was similar on each side; a Jansen- Middleton forceps was used to remove the anteroinferior two-thirds of the turbinate, generally, in two or three strips. This preserved the superior sagittally-oriented portion of the middle turbinate as a landmark, and a regular portion, posteriorly, in the region of the sphenopalatine foramen to avoid life-threatening bleeding and to guarantee the stability of the remnant middle turbinate, an approach that is shared by other authors. $4,11,14$

\section{Results}

The sample used in this study consisted of 25 people, including 11 males (44\%) and 14 females (56\%). The average age was 27.9 years old.

There was no UPSIT score difference between the groups (-Table 1).

There were no UPSIT score differences between genders (-Table 2).

\section{Discussion}

All physiological functions of the nose, including the sense of olfaction, start with air flow. The impact of middle turbinate (MT) resection on olfaction has been debated, and arguments for both improvement and impairment of olfactory sense, as a function of airflow changes, have been proposed and

Table 1 Statistical Analysis of UPSIT scores

\begin{tabular}{|l|l|l|l|l|l|l|l|}
\hline Timing & $\mathbf{N}^{\mathbf{0}}$. of cases & Mean & Median & Minimum & Maximum & SD & $\boldsymbol{p}^{*}$ \\
\hline Pre-surgery & 25 & 35.2 & 37 & 27 & 39 & 3.7 & \\
\hline Post-surgery & 25 & 35.4 & 37 & 27 & 40 & 3.5 & \\
\hline Post - Pre & 25 & 0.2 & 0 & -2 & 2 & 0.9 & 0.221 \\
\hline
\end{tabular}

Abbreviations: SD, standard deviation; UPSIT, University of Pennsylvania Smell Identification Test.

$\left.{ }^{*}\right)$ Wilcoxon rank sum test; $p<0.05$. 
Table 2 Statistical analysis according to gender

\begin{tabular}{|l|l|l|l|l|l|l|l|l|}
\hline Timing & Gender & $\mathbf{n}$ & Mean & Median & Minimum & Maximum & SD & $p$ \\
\hline Pre-surgery & Female & 14 & 36.8 & 38 & 27 & 39 & 3.2 & $0.008^{*}$ \\
\hline & Male & 11 & 33.2 & 33 & 27 & 38 & 3.5 & \\
\hline Post-surgery & Female & 14 & 36.9 & 38 & 29 & 40 & 2.9 & $0.013^{*}$ \\
\hline & Male & 11 & 33.5 & 33 & 27 & 38 & 3.5 & \\
\hline Post - Pre & Female & 14 & 0.1 & 0 & -2 & 2 & 1.0 & $0.800^{* *}$ \\
\hline & Male & 11 & 0.4 & 0 & -1 & 2 & 0.8 & \\
\hline
\end{tabular}

Abbreviation: SD, standard deviation.

$\left(^{*}\right)$ Nonparametric Mann-Whitney; $p<0.05 .\left({ }^{* *}\right)$ Analysis of covariance (ANCOVA) considering the pre-score as a covariate; $p<0.05$.

include: 1) increased airway volume following MT resection could reduce nasal resistance and increase airflow and odorant transport to the olfactory cleft; or 2) MT resection could disrupt the patterns of normal airflow that channel odorant molecules to the olfactory cleft. In general, only a small portion of the total inspiratory nasal airflow, ranging from 5-15\%, flows through the superior meatus where, theoretically, the largest area of the olfactory neuroepithelium $(\mathrm{ON})$ is located. However, the presence of $\mathrm{ON}$ in the middle turbinate has not been definitely documented. More and more the researchers from the basic area show the enormous complexity of nasal physiology. 3,5,15

Leopold et al $^{16}$ studied the relationship between bilateral human olfaction and nasal anatomy in 34 hyposmic patients (with CT and an odor identification test called odorant confusion matrix). He identified three areas influencing olfaction: i) located beneath and anterior to the cribriform plate; ii) in the space in the posterior portion of the nose and iii) below the cribriform plate. In 2002, Damm ${ }^{17}$ has analyzed normosmic subjects without nasal pathology, exploring also intranasal volumes, but with magnetic resonance imaging (MRI) and active anterior rhinomanometry. The scores found were slightly better for the right nostril and suggested a dominance of the right hemisphere; although no significant correlation was found between rhinomanometric measures and intranasal volumes, the intranasal volumes in the upper meatus (the area below the cribriform plate) and in the anterior nose, lower meatus, appear to be especially important to the sense of smell. From this last discovery, one could say that a change in a point far from the olfactory cleft can make dramatic changes in the smell ability and, on the other hand, improving the access to the superior meatus (maybe with a middle turbinectomy) could also influence positively the sense of smell.

A huge step forward was given with investigations using the technology of computational fluid dynamic (CFD), which is similar to the idea of using wind tunnels to study the automotive aerodynamic parameters (pressure, velocity, streamlines and wall shear stress), with the purposes of individualized models. ${ }^{15,18,19}$ Zhao $^{15}$ took advantage of the data from an isolated concha bullosa resection (without the variable of concurrent surgery on other portions of the nose) and with CFD analyses affirmed that, in this particular patient, partial MT resection did not impact overall nasal airflow patterns and resistance, but it did alter the regional airflow profile proximal to the osteomeatal complex. No significant changes in the uptake flux of a marked substance onto the olfactory cleft mucosa were observed post-surgery.

The distribution of the human $\mathrm{ON}$ is not entirely clear. It is thought to be situated within the olfactory cleft of the nasal cavity in the dorsal aspects of the nasal vault, septum, and superior turbinate. Biopsy studies ${ }^{9,16,20,21}$ that report sparse distribution of olfactory neurons on the septal anterior and middle portions of the MT further complicate a thorough understanding of the impact of MT resection on olfaction. Pinna $^{22}$ showed that in hematoxylin-eosin (H\&E) stained slides, ON was present in only one anterior half of the middle turbinate (from 50). According to the prevalence ratio, the odds of finding ON was 4.9 times greater in the superior turbinate than in the middle turbinate.

In conclusion, arguments for and against MT resection have been made on the basis of surgical convenience, risk of complications, postoperative care, physiologic principles and personal belief. Surgeons who favor MT resection cite improved visualization and ease of surgical antrostomy, the removal of osteitic material, decreased synechiae formation postoperatively, and ease of postoperative management. In contrast, surgeons who advocate preservation have argued that the MT is an essential surgical landmark and that its resection could contribute to a cerebrospinal fluid leak, anosmia, or frontal sinusitis, as well as disruption in nasal air conditioning, particle deposition, and airflow.

In theory, any nasal surgery could affect olfaction. Noticeably, this study has focused on the repercussion of MT resection on the olfactory function of patients free from sinusitis. The UPSIT, which is considered by many authors as the gold-standard of smell identification tests, was used as an assessment tool at a profitable moment, when a version adapted to the Brazilian population had just been published. As far we know, this is the first national study using this new version of the UPSIT and probably one of the least known in the literature in Brazil.

Another relevant point of this study is the long follow-up (average of 11 months) and the sample exclusively free from patients with sinusitis. The largest scope of data about middle resection comes from FESS, because the MT seems to be a remarkable anatomical reference. In this context, the most similar study involving UPSIT comes from Friedman, ${ }^{5}$ 
who also concluded that there was no correlation between olfaction and resection or preservation of the MT.

Two limitations of this study are: the absence of correlation of the samples resected from the MTs with immunohistochemistry, as well as the concomitant septoplasty performed. As previously mentioned, there is no consensus about the presence of olfactory epithelium in the MTs and this is already currently being addressed in a new study by the main author. And we judge that, although there are few indications for isolated middle turbinectomies, without septoplasty, it could be a way to find a more precise correlation.

We cannot recommend MT resection as a universal procedure when attempting to improve nasal patency because this proposition should be made only after a controlledrandomized study. However, we can suggest that, in selected cases, a specialist could resect the anterior two-thirds, complementary to a septoplasty (or even during FESS) in a way that is safe to olfaction.

\section{Conclusion}

This study demonstrated that partial MT resection does not influence olfaction.

Conflicts of Interests

Authors declare no conflicts of interest.

\section{References}

1 Gottarelli P. Modified inferior turbinoplasty - a new surgical approach. Milan, Italy: Springer-Verlag; 2012

2 Scheithauer MO. Surgery of the turbinates and "empty nose" syndrome. GMS Curr Top Otorhinolaryngol Head Neck Surg 2010; 9:Doc03

3 Zhao K, Scherer PW, Hajiloo SA, Dalton P. Effect of anatomy on human nasal air flow and odorant transport patterns: implications for olfaction. Chem Senses 2004;29(05):365-379

4 Clement WA, White PS. Trends in turbinate surgery literature: a 35-year review. Clin Otolaryngol Allied Sci 2001;26(02):124-128

5 Friedman M, Caldarelli DD, Venkatesan TK, Pandit R, Lee Y. Endoscopic sinus surgery with partial middle turbinate resection: effects on olfaction. Laryngoscope 1996;106(08):977-981
6 Friedman M, Tanyeri H, Landsberg R, Caldarelli D. Effects of middle turbinate medialization on olfaction. Laryngoscope 1999;109(09):1442-1445

7 Dutton JM, Hinton MJ. Middle turbinate suture conchopexy during endoscopic sinus surgery does not impair olfaction. Am J Rhinol Allergy 2011;25(02):125-127

8 Fornazieri MA, Doty RL, Santos CA, Pinna FdeR, Bezerra TFP, Voegels RL. A new cultural adaptation of the University of Pennsylvania Smell Identification Test. Clinics (Sao Paulo) 2013;68(01):65-68

9 Escada PA, Lima C, da Silva JM. The human olfactory mucosa. Eur Arch Otorhinolaryngol 2009;266(11):1675-1680

10 Godoy MDCL, Voegels RL, Pinna FdeR, Imamura R, Farfel JM. Olfaction in neurologic and neurodegenerative diseases: a literature review. Int Arch Otorhinolaryngol 2015;19(02):176-179

11 Razmpa E, Saedi B, Safavi A, Mohammadi S. Olfactory function after nasal plastic surgery. B-ENT 2013;9(04):269-275

12 Lee HY, Kim CH, Kim JY, et al. Surgical anatomy of the middle turbinate. Clin Anat 2006;19(06):493-496

13 Maniglia AJ, Maniglia JJ, Maniglia JV. Rinoplastia Estética, Funcional e Reparadora. São Paulo: Revinter; 2002:69-85

14 Banfield GK, McCombe A. Partial resection of the middle turbinate at functional endoscopic sinus surgery. J R Army Med Corps 1999; 145(01):18-19

15 Zhao K, Dalton P. The way the wind blows: implications of modeling nasal airflow. Curr Allergy Asthma Rep 2007;7(02):117-125

16 Leopold DA, Hummel T, Schwob JE, Hong SC, Knecht M, Kobal G. Anterior distribution of human olfactory epithelium. Laryngoscope 2000;110(3 Pt 1):417-421

17 Damm M, Vent J, Schmidt M, et al. Intranasal volume and olfactory function. Chem Senses 2002;27(09):831-839

18 Zhao K, Malhotra P, Rosen D, Dalton P, Pribitkin EA. Computational fluid dynamics as surgical planning tool: a pilot study on middle turbinate resection. Anat Rec (Hoboken) 2014;297(11): 2187-2195

19 Di M-Y, Jiang Z, Gao Z-Q, Li Z, An Y-R, Lv W. Numerical simulation of airflow fields in two typical nasal structures of empty nose syndrome: a computational fluid dynamics study. PLoS One 2013; 8(12):e84243

20 Apuhan T, Yildirim YS, Simşek T, Yilmaz F, Yilmaz F. Concha bullosa surgery and the distribution of human olfactory neuroepithelium. Eur Arch Otorhinolaryngol 2013;270(03):953-957

21 Féron F, Perry C, McGrath JJ, Mackay-Sim A. New techniques for biopsy and culture of human olfactory epithelial neurons. Arch Otolaryngol Head Neck Surg 1998;124(08):861-866

22 Pinna FdeR, Ctenas B, Weber R, Saldiva PH, Voegels RL. Olfactory neuroepithelium in the superior and middle turbinates: which is the optimal biopsy site? Int Arch Otorhinolaryngol 2013;17(02): $131-138$ 